\title{
Formação de carimãs e sobrevivência do bicudo do algodoeiro em cultivares de algodão
}

Formation of dry bolls and survival of the boll weevil in cotton cultivars and survival

E. B. Ribeiro ${ }^{1}$; C. A. D. da Silva'; V. R. de Novais ${ }^{3}$; W. S. do Vale ${ }^{1}$; G. dos S. Silva $^{1}$; T. L. Melo ${ }^{1}$; S. A. Leite ${ }^{1}$; M. A. Castellani ${ }^{3 *}$

${ }^{1}$ Pós-Graduação em Agronomia - Universidade Estadual do Sudoeste da Bahia - UESB, 45033-300, Vitória da Conquista - Bahia, Brasil

${ }^{2}$ Empresa Brasileira de Pesquisa Agropecuária - Embrapa Algodão, 58107-720, Campina Grande - Paraíba, Brasil

${ }^{3}$ Departamento de Fitotecnia e Zootecnia/Laboratório de Entomologia, Universidade Estadual do Sudoeste da Bahia - UESB, 45033-300, Vitória da Conquista - Bahia, Brasil

*castellani@uesb.edu.br

(Recebido em 23 de agosto de 2019; aceito em 18 de fevereiro de 2020)

O objetivo do trabalho foi estimar a formação de carimãs em diferentes cultivares de algodoeiro e a sobrevivência dos bicudos no interior dessas estruturas vegetais. A formação de carimãs foi estimada nas cultivares de algodão FM 975 WS, FM 944 GL, FM 982 GL e FM 910. Maçãs secas (carimãs) de cada cultivar foram coletadas em 20 pontos amostrais em uma área de dois hectares. Os carimãs coletados foram transportados para o laboratório e dissecados para contagem dos bicudos adultos no seu interior. A sobrevivência de adultos do bicudo foi avaliada por meio da coleta de 750 carimãs da cultivar FM 910, dissecando-se posteriormente 50 carimãs a cada 15 dias, a partir da data de coleta até 210 dias, quantificando-se adultos vivos e mortos. Os adultos foram alimentados com botões florais de Hibiscus sp. até a morte. Os resultados demonstraram que a formação de maçãs secas que não se abrem (carimãs) varia com o tipo de cultivar de algodão escolhida para plantio. A grande maioria da população de bicudos aprisionados nos lóculos dos carimãs não sobrevive por longo período de tempo, embora uma porção bastante reduzida dessa população seja capaz de sobreviver e emergir dessas estruturas por mais de cinco meses.

Palavras-chave: Anthonomus grandis, entressafra, Gossypium hirsutum.

The objective of work was to estimate the formation of dry bolls in different cotton cultivars and the survival of the cotton boll weevils inside these plant structures. The formation of dry bolls was estimated in cotton cultivars FM 975 WS, FM 944 GL, FM 982 GL and FM 910. Dried apples (dry bolls) of each cultivar were collected at 20 sample points in an area of two hectares. The collected dry bolls were transported to the laboratory and dissected to count the adult boll weevils inside. The survival of the adult boll weevil was evaluated by collecting 750 dry bolls of the cultivar FM 910, subsequently 50 dry bolls were dissected every 15 days, from the date of collection up to 210 days, quantifying live and dead adults. The adults were fed with flower buttons of Hibiscus sp. until death. The results demonstrated that the formation of dry apples that do not open (dry bolls) varies with the type of cotton cultivar chosen for planting. The big majority of the population of boll weevils inside imprisoned in the locules the dry bolls does not survive for a long time, although a very small portion of this population is able to survive and emerge from these structures for more than five months.

Keywords: Anthonomus grandis, off-season, Gossypium hirsutum.

\section{INTRODUÇÃO}

O bicudo do algodoeiro, Anthonomus grandis Boheman (1843) (Coleoptera: Curculionidae), se destaca entre as principais pragas do algodão por ocasionar perdas severas à produção. Os danos causados pelo bicudo resultam da sua alimentação nos botões florais, flores e maçãs do algodoeiro, cujas fêmeas ovipositam no interior dessas estruturas vegetais e suas larvas destroem internamente, provocando sua queda e acarretando redução da produtividade em até 87\% [1]. 
A abscisão do botão floral é ocasionada pelos compostos proteicos endopolimetilgalacturanase liberados pelas larvas de $2^{\circ}$ e $3^{\circ}$ ínstar. As flores atacadas ficam com aspecto de balão, devido à abertura anormal das pétalas e apresentam perfurações [2]. Quando as maçãs são alvo do ataque, elas se abrem irregularmente formando os carimãs que podem apresentar as fibras manchadas, aumentando a impureza do algodão e prejudicando a qualidade para comercialização [3]. Por isto, a adoção de táticas de controle para o manejo dessa praga é indispensável, tanto no período da safra como na entressafra do algodoeiro, uma vez que o bicudo pode sobreviver por vários dias sem se alimentar de plantas de algodão.

$\mathrm{Na}$ entressafra, o bicudo pode sobreviver alimentando-se de flores de outras espécies de plantas e famílias botânicas $[4,5]$ e, também, aprisionado no interior de maçãs secas de algodão (carimãs) $[6,7,8]$.

As pesquisas sobre a formação de carimãs em cultivares de algodão no Brasil são escassas, no entanto, alguns estudos realizados nos Estados Unidos demonstram que a maior parte dos bicudos sobrevive ao inverno no Arizona em maçãs e/ou carimãs dos restos de cultura a menos que estas sejam destruídas [9]. Portanto, se as operações de destruição dos restos de cultura do algodão não forem bem-sucedidas, alguns carimãs infestados pelo bicudo poderão permanecer intactos no solo, no período da entressafra e, assim, possibilitar a emergência e dispersão do inseto para áreas de refúgio $[10,8]$.

Os carimãs podem ser formados pelos danos causados pela alimentação das larvas e adultos do bicudo e oviposição de suas fêmeas nessas estruturas [11], além da alimentação de outros insetos, como o tripes que pode inocular microrganismos causadores de doenças e, por consequência de carimãs $[12,13,14]$. Dessa forma, reduzir a formação de carimãs em lavouras de algodão pode ser de fundamental importância para minimizar a infestação de bicudos na próxima safra [6, 8].

Com isso, objetivou-se determinar a ocorrência de carimãs em diferentes cultivares de algodão e avaliar a sobrevivência dos bicudos no interior dessas estruturas vegetais.

\section{MATERIAL E MÉTODOS}

Os estudos foram conduzidos entre junho e dezembro de 2014, em cultivo comercial de algodão localizado na Fazenda Tapera Grande, município de Correntina (134ㄴ $41^{\prime \prime} \mathrm{S}$; $45^{\circ}$ 46 36"O, a 849 m de altitude) região Oeste da Bahia, próxima à divisa com Goiás e no Laboratório de Entomologia da Universidade Estadual do Sudoeste da Bahia, município de Vitória da Conquista, ambos localizados no estado da BA, Brasil.

O solo é do tipo Latossolo Amarelo distrófico e a correção do $\mathrm{pH}$ e adubação foram realizadas com base na análise de solo e recomendações para a cultura, com adição de calcário (1400 kg ha $\left.^{-1}\right)$ e gesso (1200 kg ha-1) e aplicação de sulfato de amônio (320 kg ha-1), super fosfato simples $\left(600 \mathrm{~kg} \mathrm{ha}^{-1}\right)$ a lanço; bem como monofosfato de amônio $\left(100 \mathrm{~kg} \mathrm{ha}^{-1}\right)$ e cloreto de potássio (300 $\mathrm{kg} \mathrm{ha}^{-1}$ ) parcelados em duas vezes (25 dias após a emergência e 45 dias após a primeira aplicação) e ureia (300 kg ha-1), aos 30 dias após a emergência e aos 45 dias após a primeira.

No primeiro estudo, foram utilizadas as cultivares de algodão FM 975 WS; FM 944 GL; FM 982 GL e FM 910 cultivadas no campo, em área de $80.000 \mathrm{~m}^{2}$ (400 x $200 \mathrm{~m}$ ), no espaçamento de $0,90 \times 0,20 \mathrm{~m}$, tendo-se deixado uma planta por cova após o desbaste. Essas cultivares foram escolhidas por apresentarem ciclo semelhante, produtividade média superior a 300 arrobas ha ${ }^{-1}$ de algodão em caroço e por serem as mais plantadas pelos produtores brasileiros no bioma Cerrado. No entanto, elas diferem em relação à tecnologia transgênica, onde GL (Glitol/Liberty Link®) apresenta resistência aos herbicidas glifosato e glufosinato de amônio, enquanto WS (WideStrike ${ }^{\circledR}$ ) apresenta resistência aos principais lepidópteros-praga do algodoeiro e contém as proteínas tóxicas Cry1Ac, Cry1F e Vip3A do Bacillus thuringiensis incorporadas ao genoma das plantas. A cultivar FM 910 é convencional, não transgênica.

Utilizou-se delineamento inteiramente casualizado, pois as características físico-químicas do solo, localização das parcelas e tratos culturais utilizados eram bastante homogêneas. Foram utilizados quatro tratamentos, representados pelas cultivares de algodão. Os carimãs de cada cultivar foram coletados em 20 pontos amostrais, totalizando 20 repetições. Em cada ponto, coletaram-se todos os carimãs de 20 plantas na mesma linha, em uma área de dois hectares $(100 \mathrm{~m}$ x 200m). Os carimãs foram levados ao laboratório, colocados em sacos de papel Kraft, 
individualizados por tratamento e acondicionados em sacos de nylon à temperatura de $25 \pm 2^{\circ} \mathrm{C}$ até o momento das avaliações. Em cada avaliação procedeu-se a abertura manual dos lóculos de cada carimã para contagem dos bicudos adultos presentes no seu interior. Foi determinado o número médio de carimãs com adultos do bicudo e de bicudos vivos e mortos por carimã. Os tratos culturais e fitossanitários utilizados para condução da lavoura foram os mesmos para as quatro cultivares durante todo o período de produção.

Um segundo experimento, para se determinar a sobrevivência de adultos do bicudo no interior de carimãs, foi conduzido utilizando-se um total de 750 carimãs de algodão da cultivar FM 910, em função do histórico de altas infestações dessa praga nessa cultivar. Os carimãs foram coletados no final do ciclo da cultura, quando o algodão já estava apto para a colheita. A amostragem, coleta e acondicionamento dos carimãs foram realizados conforme descrito no primeiro experimento. A avaliação da sobrevivência foi realizada a cada 15 dias ao longo de 210 dias, retirando-se uma amostra de 50 carimãs para dissecação, contagem e separação dos adultos vivos e mortos. Os adultos vivos foram transferidos e mantidos em gaiolas do tipo baleiro, que consiste em recipiente plástico transparente $(22 \mathrm{~cm} \times 13 \mathrm{~cm} \times 13 \mathrm{~cm})$, com tampa larga e tecido voil na lateral, e alimentados com botões florais de Hibiscus sp., em temperatura ambiente em torno de $25 \pm 2{ }^{\circ} \mathrm{C}$ até a morte natural.

Os dados obtidos foram submetidos a testes de normalidade e homocedasticidade, e submetidos ao teste da ANOVA para posterior comparação de médias pelo teste Tukey $(\mathrm{p}<0,05)$ pelo Programa R [15].

\section{RESULTADOS E DISCUSSÃO}

O número médio de carimãs variou entre as cultivares ( $\mathrm{p}<0,05)$, destacando-se a FM 910 com o maior número $(51,80)$, diferindo significativamente das demais; seguida da cultivar FM 982 GL $(33,45)$ que apresentou número médio de carimãs intermediário entre a FM 910 e as demais e, por fim, as cultivares FM 944 GL $(23,35)$ e FM 975 WS $(22,95)$ que não diferiram entre si e que apresentaram as menores médias de carimãs (Figura 1).

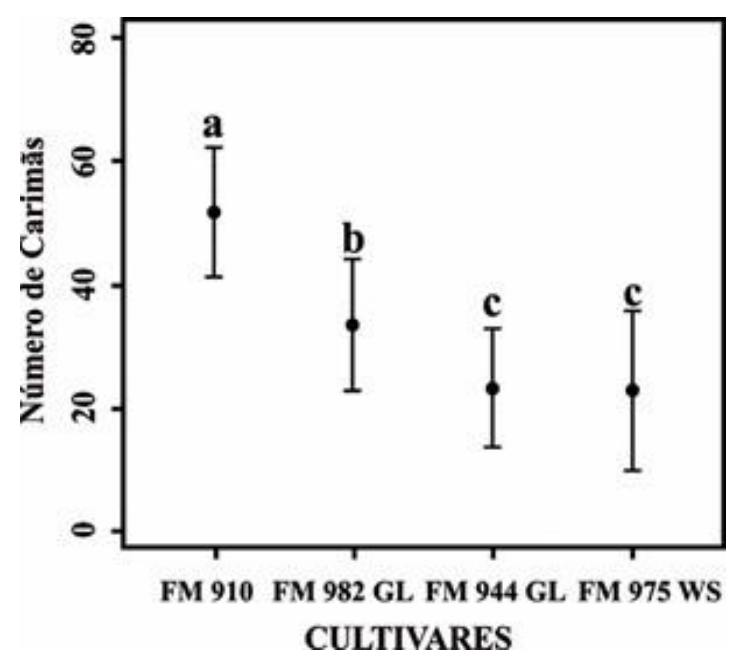

Figura 1: Número médio de carimãs nas cultivares FM 910, FM 982 GL, FM 944 GL e FM 975 WS. Médias seguidas pela mesma letra não diferem pelo teste Tukey $(p<0,05)$.

Foram obtidos bicudos adultos em carimãs de todas as cultivares. Dentre as cultivares avaliadas, a quantidade de carimãs com adultos foi maior na cultivar FM $910(8,60)$, diferindo significativamente demais cultivares ( $\mathrm{p}<0,05)$, as quais não diferiram entre si para essa variável (FM 982 GL = 1,40; FM 944 GL = 1,25; e FM 975 WS = 4,25) (Figura 2A).

Adultos vivos do bicudo foram obtidos em todas as cultivares. Dentre os carimãs examinados a cultivar com o maior número médio de indivíduos vivos foi a cultivar FM 910, diferindo significativamente das demais cultivares (Figura 2B). Esses maiores números de carimãs, carimãs com adultos e adultos vivos observados na cultivar de algodão FM 910, se deve, provavelmente, ao fato desta cultivar ser convencional (não transgênica). Algodoeiros transgênicos podem diferir 
de suas contrapartes não transgênicas em algumas características agronômicas e fisiológicas [16, 17, 18], assim como, na sua capacidade de hospedar fungos endofíticos nas folhas [19]. Do mesmo modo, algodoeiros convencionais submetidos ao ataque de bicudos adultos podem diferir em relação à quantidade de capulhos formados [20].

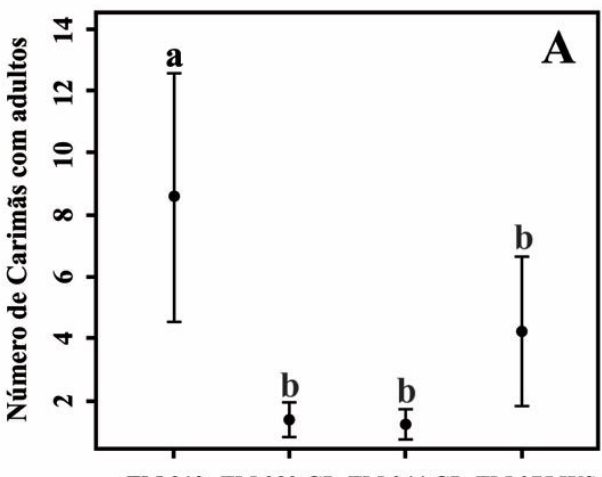

FM 910 FM 982 GL FM 944 GL FM 975 WS

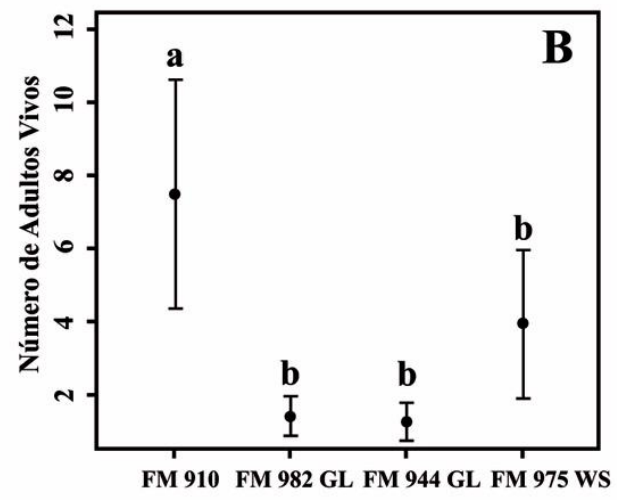

FM 910 FM 982 GL FM 944 GL FM 975 WS

CULTIVARES

Figura 2: Número médio de carimãs com adultos e número médio de adultos vivos coletados nas cultivares FM 910, FM 982 GL, FM 944 GL e FM 975 WS de algodão. Médias seguidas pela mesma letra não diferem pelo teste Tukey $(p<0,05)$.

No que diz respeito à sobrevivência de adultos do bicudo no interior de carimãs da cultivar de algodão FM 910, observou-se a presença de bicudos adultos em 28 carimãs de um total de 750 carimãs avaliados $(3,7 \%)$ e, dentre estes, apenas 10 carimãs (1,3\%) apresentaram adultos vivos, totalizando 14 bicudos vivos até a última avaliação (Tabela 1). Essas taxas de bicudos vivos observadas no presente trabalho foram inferiores às taxas de $20 \%$ e $4 \%$ encontradas por Bariola et al. (1984) [6] em carimãs de algodoeiros cultivados no Arizona nos anos de 1982 e 1983, respectivamente. Tal fato pode ser atribuído às diferenças metodológicas entre os experimentos, pois, enquanto Bariola et al. (1984) [6] avaliaram somente os carimãs que possuíam orifício de alimentação e/ou postura, neste trabalho, os carimãs foram dissecados aleatoriamente, independente da presença ou não destas perfurações.

Tabela 1: Números de carimãs, carimãs com adulto, carimãs com adultos vivos e sobrevivência dos adultos alimentados com pólen de Hibiscus sp. dentro e foram de carimãs da cultivar de algodão FM 910 em quinze épocas de avaliação. Vitória da Conquista, BA, 2014.

\begin{tabular}{|c|c|c|c|c|c|c|c|}
\hline \multirow{2}{*}{$\begin{array}{c}\text { Época } \\
\text { de } \\
\text { avaliaçã } \\
\text { o (Dias) } \\
\end{array}$} & \multicolumn{2}{|c|}{$\begin{array}{r}\text { Carimã } \\
\text { com adulto }\end{array}$} & \multicolumn{2}{|c|}{$\begin{array}{c}\text { Carimã } \\
\text { com adulto } \\
\text { vivo }\end{array}$} & \multirow{2}{*}{$\begin{array}{c}\begin{array}{c}\text { Adult } \\
\text { o vivo }\end{array} \\
n^{\circ} \\
\end{array}$} & \multirow{2}{*}{$\begin{array}{c}\begin{array}{c}\text { Sobrevivênci } \\
\text { a fora do } \\
\text { carimã }\end{array} \\
\text { Dias } \\
\end{array}$} & \multirow{2}{*}{$\begin{array}{c}\begin{array}{c}\text { Sobrevivênci } \\
\text { a total }\end{array} \\
\text { Dias }\end{array}$} \\
\hline & $\mathbf{n}^{\circ}$ & $\%$ & $\mathbf{n}^{\circ}$ & $\%$ & & & \\
\hline 0 & 5 & 10 & 1 & 2 & 1 & 41 & 41 \\
\hline 15 & 3 & 6 & 3 & 6 & 6 & $\begin{array}{l}43,44,47,50 \\
\quad 51 \text { e } 55\end{array}$ & $\begin{array}{c}58,59,62 \\
65,66 \text { e } 70\end{array}$ \\
\hline 30 & 2 & 4 & 2 & 4 & 3 & 9,11 e 12 & $39,41,42$ \\
\hline 45 & 2 & 4 & 2 & 4 & 2 & 13 e 15 & 58 e 60 \\
\hline 60 & 2 & 4 & 1 & 2 & 1 & 35 & 95 \\
\hline 75 & 3 & 6 & 0 & - & 0 & - & - \\
\hline 90 & 1 & 2 & 0 & - & 0 & - & - \\
\hline 105 & 0 & - & 0 & - & 0 & - & - \\
\hline 120 & 0 & - & 0 & - & 0 & - & - \\
\hline 135 & 1 & 2 & 1 & 2 & 1 & 20 & 155 \\
\hline 150 & 3 & 6 & 0 & $=$ & 0 & - & - \\
\hline 165 & 0 & - & 0 & $=$ & 0 & - & - \\
\hline 180 & 2 & 4 & 0 & $=$ & 0 & - & - \\
\hline
\end{tabular}




\begin{tabular}{cccccccc}
\hline 195 & 3 & 6 & 0 & $=$ & 0 & - & - \\
210 & 1 & 2 & 0 & $=$ & 0 & - & - \\
\hline \multirow{2}{*}{ Total } & 2 & 3, & 1 & 1, & \multirow{2}{*}{14} & \multirow{2}{*}{ Média $=31,9$} & \multirow{2}{*}{ Média $=65,1$} \\
\hline
\end{tabular}

Os números médios de carimãs com bicudos e com adultos vivos variaram entre as épocas de avaliação, com a maior parte deles concentrados nos primeiros 75 dias $(60,7 \%)$ e 60 dias $(90,0 \%)$, respectivamente. A longevidade média dos bicudos sobreviventes fora dos carimãs variou de nove até 55 dias, com média de 31,9 dias, enquanto que no interior de carimãs foi de 65,1 dias, com variação de 39 a 155 dias, quando se constatou o último bicudo vivo (Tabela 1). Tal resultado corrobora com aquele observado por Pires et al. (2017) [21] que encontraram um adulto vivo do bicudo emergido de carimã em fevereiro de 2013, ou seja, 153 dias após a incubação, nos capulhos coletados ao final do ciclo de cultivo do algodoeiro em 2012. Segundo esses autores isso representou $0,25 \%$ dos bicudos da população IV e $0,0002 \%$ dos bicudos das cápsulas de todas as quatro populações amostradas (5.544 adultos), um número que não é desprezível, considerando o alto potencial biótico do inseto.

Os resultados obtidos nesta pesquisa indicam que a maioria dos bicudos não é capaz de sobreviver por longos períodos de tempo abrigados no interior de carimãs, provavelmente pela limitação de espaço e ausência de alimento e, portanto, podem ser controlados de forma eficiente por meio das práticas recomendadas de destruição de restos de cultura e soqueira, assim como, pelo uso de cultivares de algodão menos propensas à formação de carimãs, a exemplo das cultivares FM 944 Gl e FM 975 WS. Por outro lado, novas pesquisas devem realizadas para compreender porque uma porção bastante reduzida dessa população de bicudos adultos é capaz de sobreviver e emergir de carimãs após um período superior a cinco meses.

\section{CONCLUSÃO}

A formação de maçãs secas que não se abrem (carimãs) varia com o tipo de cultivar de algodão escolhida para plantio.

A grande maioria da população de bicudos aprisionados nos lóculos dos carimãs não sobrevive por longo período de tempo, embora uma porção bastante reduzida dessa população seja capaz de sobreviver e emergir dessas estruturas por mais de cinco meses.

\section{AGRADECIMENTOS}

À Universidade Estadual do Sudoeste da Bahia (UESB) pelo apoio e à Fapesb pela concessão da bolsa de estudos concedida ao primeiro autor e à Fazenda Tapera Grande e toda Família Orth pela disponibilização da área de cultivo para realização dos experimentos, bem como pela estadia.

\section{REFERÊNCIAS BIBLIOGRÁFICAS}

1. Silva CAD, Ramalho FS. Kaolin spraying protects cotton plants against damages by boll weevil Anthonomus grandis Boheman (Coleoptera: Curculionidae). J Pest Sci. 2013;86(2):563-569, doi.org/10.1007/s10340-013-0483-0.

2. Almeida RP, Silva CAD, Ramalho FS. O agronegócio do algodão no Brasil. In: Beltrão NEM, Azevedo DMP. (eds) Manejo integrado de pragas do algodoeiro no Brasil. Brasília: Embrapa Informação Tecnológica, 2008. p. 1034-1098.

3. Azambuja R, Degrande PE. Trinta anos do bicudo-do-algodoeiro no Brasil. Arq Inst. Biol. 2014;81:377410, doi.org/10.1590/1808-1657000012013.

4. Ribeiro PA, Sujii ER, Diniz IR, Medeiros MA, Salgado-Labouriau ML, Branco MC, Pires CS, Fontes EM. Alternative food sources and overwintering feeding behavior of the boll weevil, Anthonomus grandis Boheman, (Coleoptera: Curculionidae) under the tropical conditions of Central Brasil. Neotrop Entomol. 2010;39(1):28-34, doi.org/10.1590/S1519-566X2010000100005.

5. Macêdo JA., Castellani MA, Santos F de AR dos, Oliveira PP, Pérez-Maluf R. Fontes alternativas de pólen utilizadas pelo bicudo-do-algodoeiro em duas regiões produtoras de algodão na Bahia. Rev Caatinga. 2015;28(3):255-262, doi.org/10.1590/1983-21252015v28n329rc. 
6. Bariola LA, Henneberry TJ, Bergman D. Boll weevils (Coleoptera: Curculionidae): emergence from bolls during the spring, and trapping adults in Arizona. J Econ Entomol. 1984;77(5):1166-1170, doi.org/10.1093/jee/ 77.5 .1166$.

7. Showler AT. Subtropical boll weevil ecology. Amer Entomol. 2007;53(4):240-249, doi.org/10.1093/ae/53.4.240.

8. Ribeiro EB, Castellani MA, Silva CAD da, Melo TL, Silva G dos S, Vale WS do, Santos AS. Métodos de destruição de restos de cultura do algodoeiro e sobrevivência do bicudo. Pesq Agropec Bras. 2015;50(11):993-998, doi.org/10.1590/S0100-204X2015001100001.

9. Fye RE, Leggett JE, Bonham CD. Winter survival of boll weevil complex in Arizona. J Econ Entomol. 1970;63:1071-1074, doi.org/10. 1093/jee/62.1.147.

10. Leggett JE, Fye RE. The role of moisture in the winter survival of the boll weevil complex in Arizona. J Econ Entomol. 1969;62(1):147-149, doi.org/10.1093/jee/62.1.147.

11. Santos, R.C.; Marcellino, L.H.; Monnerat, R.G.; Gander, E.S. (2003). Mechanical damage in cotton buds caused by the boll weevil. Pesq Agropec Bras. 2003;38(11):1351-1355, doi.org/10.1590/S0100204X2003001100015.

12. Mailhot DJ. Influence of flower Thrips on Fusarium hardlock severity. Plant Disease. 2007;1:14231429, doi.org/10.1094/PDIS-91-11-1423.

13. Osekre EA, Wright DL, Marois JJ, Funderburk J. Flower-Inhabiting Frankliniella Thrips (Thysanoptera: Thripidae), Pesticides, and Fusarium Hardlock in Cotton. J Econ Entomol. 2009;102:887-896, doi.org/ 10.1603/029.102.0305.

14. Srivastava P, Mailhot DJ, Leite B, Marois JJ, Wright DL, Nichols LR. Fusarium verticillioides (Saccardo) Nirenberg Associated with Hardlock of Cotton. Curr Microbiol. 2010;61(2):79-84, doi.org/10.1007/s00284-009-9578-5.

15. R Core Team. R: A language and environment for statistical computing. R Foundation for Statistical Computing, Vienna, Austria. 2018, www.R-project.org/.

16. Elmore RW, Roeth FW, Nelson LA, Shapiro CA, Klein RN, Knezevic SZ, Martin A. Glyphosateresistant soybean cultivar yields compared with sister lines. Agron J. 2001;93(2):408-412, doi.org/10.2134/agronj2001.932408x.

17. Dong H, Li WJ, Tang W, Li ZH, Zhang DM. Effects of genotypes and plant density on yield, yield components and photosynthesis in Bt transgenic cotton. J Agron Crop Sci. 2006;192:132-139, doi.org/ 10.1111/ j.1439-037X.2006.00196.x.

18. Wang F, Peng S, Cui K, Nie L, Huang J. Field performance of Bt transgenic crops: A review. Austral. J Crop Sci. 2014;8(1):18-26.

19. Vieira PDS, Souza-Motta CM, Lima DR, Torres JB, Quecine MC, Azevedo JL, Oliveira NT. Endophytic fungi associated with transgenic and non-transgenic cotton. Mycology. 2011;2(2):91-97, doi.org/10.1080/ 21501203.2011 .584390 .

20. Abreu SC, Resende M, Rios S, Alvarenga C, Gonçalves N. Desempenho de genótipos de algodoeiro sob pressão de bicudo. Rev Biot. 2013;26(2): 78-82, doi.org/10.5007/2175-7925.2013v26n2p77.

21. Pires CSS, Pimenta M, Mata RA, Souza LM, Paula DP, Sujii ER, Fontes EMG. Survival pattern of the boll weevil during cotton fallow in Midwestern Brazil. Pesq Agropec Bras. 2017;52(3):149-160, doi.org/10.1590 /S0100-204X2017000300002. 